\title{
L'application concrète du développement durable : Les écovillages peuvent-ils relever le défi?
}

\author{
Prudence-Élise Breton \\ University of Northern British Columbia
}

\section{Introduction}

Bien qu'une certaine prise de conscience commence à poindre à l'horizon à l'effet qu'il faille adopter des mesures pour atténuer la dégradation des ressources naturelles, l'analyse de l'empreinte écologique ${ }^{1}$ de notre mode de vie individualiste indique qu'il y a encore loin de la coupe aux lèvres. Les banlieues, mais aussi de nombreuses petites localités périurbaines dont le modèle d'aménagement imite ces dernières, illustrent avec éloquence ce mode de vie. Il s'agit, en définitive, de choix individuels qui fragilisent la pérennité de la vie sur Terre.

En effet, on observe dans la tendance dominante de l'étalement urbain, caractérisée par le «navettage » des travailleurs vers la ville ${ }^{2}$, une évidente segmentation spatiale entre travail, habitation, commerces et loisirs, ce qui induit
L'adoption du mode de vie banlieusard a quelque chose de séduisant qu'aucune autre alternative ne semble pouvoir égaler. que chose de séduisant qu'aucune autre alternative ne semble pouvoir égaler. En effet, il a le mérite incontestable d'être, pour bon nombre de gens, un heureux compromis qui leur permet de répondre simultanément à plusieurs besoins importants. D'un côté, il répond aux besoins de sécurité et de détente, grâce à un espace vert privé procurant intimité et tranquillité, ce que les logements contigus de la ville n'offrent pas toujours. D'autre part, au niveau professionnel, ce mode de vie facilite l'épanouissement des individus par une autonomie et une flexibilité de déplacement que rend possible le transport automobile.

En attendant que des politiques d'aménagement territorial et de transport apportent des correctifs appropriés, il faut toutefois mettre en branle des interventions sur le terrain, afin d'expérimenter une autre façon de vivre qui réde nombreux problèmes sociaux et écologiques. Parmi les impacts écologiques, mentionnons qu'une plus grande utilisation de l'automobile entraîne une surconsommation des espaces agricoles et naturels. De plus, le transport routier représente $35 \%$ des émissions de gaz à effet de serre du secteur des transports ou environ le tiers des émissions totales ${ }^{3}$, devançant le secteur industriel, qui représente $31 \%$ des émissions totales. Le secteur des transports est donc sans contredit le principal responsable des émissions polluantes dans l'atmosphère. De plus, ce mode de vie segmenté ne favorise ni l'entraide entre voisins ni le développement d'activités locales diversifiées, tant communautaires, sociales, qu'économiques.

Malgré toutes ces conséquences, le modèle ne semble pas présenter d'indice d'essoufflement, bien au contraire. L'adoption du mode de vie banlieusard a quel- ponde à nos besoins mais ce, dans le cadre d'un mode de vie respectueux de l'environnement et de ses écosystèmes. C'est essentiellement ce que propose le mouvement des écovillages.

\section{Historique et définition du concept d'écovillage}

La Déclaration adoptée par les dirigeants de 178 pays réunis à Rio de Janeiro en 1992 pour discuter de l'avenir de la planète décrétait: "Les êtres humains ont droit à une vie saine et productive en harmonie avec la nature». En regard de cet énoncé, le fastidieux Agenda 21 s'en est suivi, fixant des objectifs à atteindre, dont l'un fut proposé par Gaia Trust of Danemark $^{4}$ et Global Ecovillage network (GEN) $)^{5}$, afin de promouvoir le développement d'exemples concrets de communautés viables pouvant servir de laboratoires 
élaborant et mettant en œuvre des solutions stratégiques, techniques, pratiques et à dimension humaine face à certains des problèmes soulevés par le rapport Brundtland. Ces exemples pourraient servir de champs expérimentaux reproductibles à travers le monde, autant en zone urbaine que rurale, et dans divers types d'habitats, de climats et de cultures. Le concept d'écovillage était officiellement né.

Un écovillage ou écohameau (plus petite dimension) est un mode d'habitation collectif qui tente de répondre aux impératifs écologiques de l'occupation du territoire par l'être humain (densification des habitats, utilisation de matériaux locaux et naturels, économie d'énergie, mise sur pied de micro-entreprises locales...). Préoccupés par les problèmes écologiques, les occupants et bâtisseurs de ces habitats recherchent la proximité de personnes animées des mêmes valeurs et optent généralement pour une gestion participative et des prises de décision par consensus. Trois cents de ces communautés ${ }^{6}$ ont déjà été répertoriées à travers le monde et, bien qu'elles puissent se révéler assez différentes les unes des autres, on définit globalement les écovillages comme des communautés engagées à vivre de manière viable écologiquement, économiquement, culturellement et spirituellement ${ }^{7}$. L'émergence des écovillages représente donc une volonté innovante $d$ 'opérationnalisation du développement durable.

\section{Démarche pour l'étude de cas}

Les données factuelles sur le sujet étant peu nombreuses (il n'y a pas encore d'écovillage comme tel au Québec), j'ai opté pour une collecte de données réalisée par des visites auprès de quelques initiatives ciblées. J'ai ainsi entrepris un voyage exploratoire dans trois communautés américaines, dont deux sont considérées comme des écovillages. Le projet, supporté financièrement par l'Office Québec-Amérique pour la Jeunesse (OQAJ), consistait en une immersion dans les activités quotidiennes des habitants et la réalisation d'entrevues. Pour encadrer cette démarche et structurer la cueillette de données, une grille d'évaluation du développement durable élaborée par le professeur Claude Villeneuve de 'UQAC $^{8}$ a été utilisée. Quoique qualitatif, le résultat obtenu se veut toutefois mesurable et permet une certaine comparaison entre les communautés visitées.
La collecte de données a été effectuée auprès de trois communautés. D'une part, l'échantillonnage nonprobabiliste était constitué de deux écovillages : Sirius (Shutesbury, Massachusette) et Earthaven (Black Mountain, Caroline du Nord). D'autre part, une troisième communauté, Ganas (dans la ville de New York), tenait en quelque sorte lieu de groupe témoin; n'ayant pas d'ambition ou d'objectif écologique spécifique, elle appartient néanmoins à la famille des communautés intentionnelles ${ }^{9}$. L'évaluation de cette communauté, effectuée selon les mêmes critères que les deux écovillages, permet d'isoler la variable « vie en communauté », afin d'assurer que d'éventuels pointages obtenus par les écovillages pour le pôle écologique ne soient pas uniquement le fait d'une vie commune, mais plutôt le fruit d'efforts spécifiques effectués en ce sens.

\section{Un écovillage ou écohameau (plus petite dimension) est un mode d'habitation collec- tif qui tente de répondre aux impératifs éco- logiques de l'occupation du territoire par l'être humain.}

Afin d'identifier les éléments de la grille, tout en dégageant le sens que les acteurs donnent à leur choix et actions, l'observation participative a été la méthode principalement utilisée pour la collecte des données. À chaque endroit, une visite guidée et commentée par l'un des habitants a été faite, suivie d'une seconde visite, effectuée seule pour prendre photos et notes. Chacune des communautés m'a donné accès à une ou plusieurs réunions de prise de décision. J'ai également pris personnellement part à leurs activités et tâches communautaires journalières (repas, entretien ménager, jardinage, construction écologique, entretien du terrain) et ponctuelles (fêtes, formations, soirées d'animation). Ces occasions permettaient, au cours d'échanges informels, d'obtenir des informations sur divers aspects du fonctionnement et des infrastructures ainsi que sur les valeurs de la communauté et de ses habitants. Finalement, des entrevues semi-dirigées réalisées auprès de quelques habitants sont venues compléter certaines informations, vérifier des faits et obtenir des éclaircissements nécessaires pour compléter la grille d'analyse. 


\section{L'outil de mesure du développement durable}

Élaborée par le professeur Claude Villeneuve de l'UQAC, la grille d'analyse comporte quatre pôles : économique, social, écologique et équité. Chacun d'eux se décline en lignes directrices (de 4 à 6 par pôle), puis en objectifs (de 1 à 8 par ligne directrice), qui sont pondérés, puis notés; les résultats sont ensuite additionnés pour obtenir le score de chaque pôle. Enfin, l'utilisateur peut appuyer ses observations par des plages de justification et des pistes de bonification, qui incitent à un questionnement sur la manière de mieux atteindre l'objectif. (Voir l'exemple au tableau 1) Les scores finaux de chaque pôle sont ensuite compilés et illustrés à l'aide d'un tétraèdre (voir figure 1). La démarche a été fortement inspirée par cette grille d'analyse mais son utilisation a été légèrement modulée en fonction de certaines contraintes de temps, d'argent et d'organisation. (Voir encadré $1)$.

\section{Encadré 1}

\section{Ajustements apportés à l'utilisation de la grille de développement durable dans le cadre de cette étude}

Cet outil doit être habituellement utilisé de façon longitudinale, c'est-à-dire qu'il doit servir à comparer un même projet à l'intérieur de certains repères dans le temps. Cependant, la nature de la démarche, qui se voulait ponctuelle (exploration en vue d'un projet d'étude de maîtrise) m'a plutôt amené à entreprendre une étude comparative de cas ciblés, choisis pour leur longévité et la reconnaissance dont ils bénéficient dans le milieu des écovillages.

La pondération doit se faire de concert avec les acteurs en cause. Toutefois, l'établissement d'un tel consensus n'était pas réaliste, avec trois communautés situées dans trois états américains différents et qui représentent environ 175 personnes au total. Par conséquent, la pondération a été élaborée de façon parallèle aux observations et constats faits sur le terrain. J'ai par la suite sollicité le concours de David Connell, professeur pour le programme de maitrise en Ressources naturelles et environnement de l'University of Northern British Columbia, qui porte un intérêt marqué pour toute forme de communauté intentionnelle, notamment les écovillages. À la suite de divers échanges, nous en sommes finalement venus à un consensus sur la pondération.

Finalement, le score final de chaque pôle est normalement exprimé sur une échelle de 0 à 1 . Or, dans le cadre de cette démarche, les scores ont plutôt été reportés en pourcentages, afin de mettre les constats en lumière de façon plus différenciée, notamment dans le tétraèdre.

\section{Présentation des trois communautés}

Le voyage s'est déroulé en trois temps. D'abord, la première visite, d'une durée de cinq jours, a eu lieu dans la communauté de Ganas ${ }^{10}$, située sur l'île de Staten Island dans la ville de New York. Fondée en 1978, cette organisation met l'emphase non pas sur l'écologie ou le développement durable mais bien sur les communications et les relations interpersonnelles. La communauté compte dix maisons logeant environ 90 habitants et des locaux commerciaux occupés par quatre entreprises gérées par les membres de la communauté.
Dans un deuxième temps, le voyage s'est poursuivi par une immersion de quatorze jours dans la communauté de Sirius ${ }^{11}$, située dans le village de Shutesbury au Massachusette. Les 35 écovillageois habitent dans l'un des quatre bâtiments résidentiels bordant une terre de 90 acres, dont Sirius a fait l'acquisition il y a 28 ans.

Finalement, l'expérience s'est terminée par un séjour de deux semaines à Earthaven ${ }^{12}$, un écovillage situé à Black Mountain en Caroline du Nord. Il a été mis sur pied en 1995, sur un terrain montagneux de 320 acres. Déjà, 50 habitants prennent part à cet ambitieux projet prévu pour 150 personnes. 


\section{Résultats de la grille d'analyse}

\section{Faits saillants}

Cette première partie consiste à faire ressortir les éléments les plus contrastés et les plus marquants qui se dégagent du tétraèdre des communautés (voir figure 1). Cette étape, qui se situe au niveau des scores obtenus pour les trois communautés quant aux quatre pôles, guidera le choix d'objectifs spécifiques de la grille qui seront ensuite approfondis dans l'analyse spécifique.
À prime abord, le tétraèdre permet de constater que le pôle écologique de Ganas, le groupe témoin, présente un net écart par rapport aux deux écovillages. Son score, qui se situe au dernier rang, avec 47,4 \%, montre que la vie en communauté ne suffit pas à elle seule pour répondre aux critères écologiques du développement durable. Ainsi, les efforts de Sirius et Earthaven en la matière semblent effectivement leur permettre de s'approcher davantage de cet idéal, tel que le démontre d'emblée le relatif équilibre entre les quatre pôles. La deuxième partie de l'analyse permettra d'examiner ce pôle pour les trois communautés.

\section{Figure 1}

\section{Score des communautés}

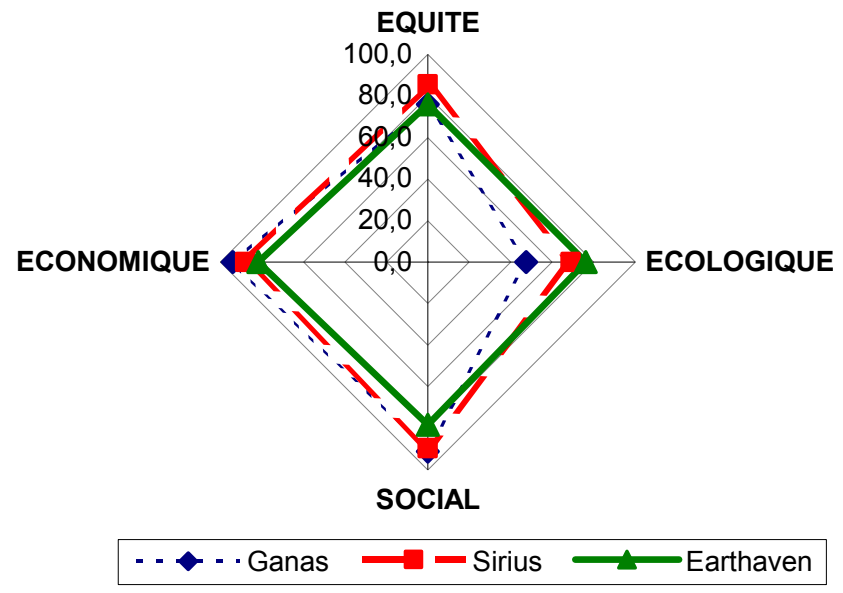

Par ailleurs, il apparaît important de souligner ici un constat marquant du tétraèdre et qui se révèle pour le moins surprenant. En effet, on remarque que le pôle écologique ressort globalement avec le plus faible pointage et ce, même pour les deux écovillages. Comment se fait-il que des écovillages ayant comme but ultime le développement durable et mettant a priori l'emphase sur l'écologie n'ont pas un meilleur résultat en la matière? Bien qu'aucune réponse immédiate ne puisse être apportée, plusieurs éléments peuvent être évoqués afin de tenter d'expliquer ce paradoxe. Ainsi, y aurait-il des barrières administratives ou réglementaires dans la macrostructure (état, comté, municipalité) entravant la mise en place de certaines infrastructures écologiques? Un apport financier additionnel serait-il nécessaire pour un développement plus étendu de technologies innovatrices dans ce contexte expérimental ? Les tendances de comportement individualiste et de surconsommation (donc, non durable) du système dominant persisteraient-elles significativement dans ces micro-sociétés qui ne sont, et avec raison, pas hermétiques? Quoi qu'il en soit, ce constat indique que les objectifs du pôle écologique prescrits par le développement durable représentent un défi de taille à relever.

Ensuite, ces trois communautés, dont l'existence est principalement motivée par des objectifs de nature sociale ou écologique, atteignent des résultats souvent meilleurs au niveau du pôle économique. Ce constat, qui peut paraître étonnant à prime abord, s'explique toutefois en bonne partie par la nature même des objectifs du pôle économique de la grille d'analyse. En fait, ceux-ci concernent le partage et la redistribution de la richesse, la mise en commun des connaissances, la qualité et la durabilité des biens produits, etc. Ces éléments traduisent le sens profond du terme " économie », soit répondre aux besoins de tous les indivi- 
dus, et non pas le sens qu'on lui donne couramment, qui correspond essentiellement à la notion de «profit ». Ainsi, Ganas obtient le score le plus élevé pour le pôle économique avec 93,9 \% qui de par sa localisation en plein cœur d'une ville populeuse et très dense, est avantagée par les économies d'échelle. Il sera par ailleurs question des autres facteurs contribuant à ce score élevé dans l'analyse spécifique.

Par ailleurs, malgré le résultat plus élevé pour le pôle écologique de la courbe de Earthaven, on remarque que le score de cet écovillage est légèrement plus bas pour les trois autres pôles. Son pointage arrive derrière celui de Sirius et à égalité avec celui de Ganas pour le pôle équité et se classe un peu moins bien pour le pôle économique; de plus, il accuse un écart appréciable pour le pôle social. Ce dernier pôle sera subséquemment examiné de plus près.

Finalement, le dernier grand constat qui guidera l'analyse spécifique est le premier rang occupé par le pointage de Sirius pour le pôle équité. Cet écovillage devance en effet les deux autres communautés par près de dix points de pourcentage (respectivement $85,5 \%$ et $76 \%$ pour Ganas et Earthaven). Les raisons de ce classement seront abordées plus en profondeur ci-dessous.

\section{Analyse spécifique}

Dans le cadre de cette section, certains objectifs précis pour chacun des cas seront décrits. Vu la quantité considérable de données recueillies pour les trois communautés grâce à la grille d'analyse, je ne présenterai pour Ganas, Sirius et Earthaven que des extraits de la grille d'analyse pour deux des pôles, le tout précédé d'une courte mise en contexte. L'analyse exposera donc, pour chaque cas, un objectif d'une des lignes directrices, et ce, pour deux des quatre pôles. Cet objectif inclura la ligne directrice, sa pondération, sa note, sa justification et sa piste de bonification (voir tableau 1 pour un exemple). Pour chacune des communautés, un objectif du pôle écologique sera choisi et examiné ainsi qu'un autre objectif dans un autre pôle, qui servira à expliciter les contrastes soulignés en première étape d'analyse. Les objectifs seront choisis en fonction de leur potentiel à expliquer le fort ou faible score pour le pôle concerné.

\section{Ganas}

Cette communauté est située sur Staten Island, un quartier pauvre de la ville de New York. Les quelques 90 habitants se partagent dix maisons avec chacune leur cuisine et un garde-manger bien rempli. La dimension et le prix des chambres varient et elles sont pourvues d'une salle de bain privée ou partagée. Deux bâtiments comprennent des pièces et des équipements communs (cuisine communautaire, salle à manger, salon, ordinateurs, etc.), en plus d'une cour extérieure avec piscine, terrasse, jardin et terrain de jeu.

Les quatre entreprises gérées par Ganas ont en commun de récupérer, recycler et revendre diverses marchandises; elles sont connues sous la bannière «Every Thing Goes». Il s'agit d'une friperie, d'un magasin de meubles usagés, d'un magasin de meubles antiques et d'un café-boutique de livres usagés qui embauchent en tout 15 personnes de la communauté à temps plein ou partiel.

Le score élevé de Ganas pour le volet économique s'explique inexorablement par le partage et la mise en commun des biens et des infrastructures, ce qui permet une répartition de la richesse parmi les membres. Certains, moins fortunés, ne pourraient possiblement pas bénéficier d'autant de biens, si ce n'était de leur choix de vivre en communauté. De plus, les entreprises sont autant de façons de s'ouvrir sur la population locale en leur offrant à bas prix des biens semiessentiels, tels de l'ameublement, des vêtements et des livres. L'objectif 1.2 permet d'illustrer ce fait (voir tableau 1). 
Tableau 1 :

Ganas - Extrait du pôle économique :

répondre aux besoins matériels du plus grand nombre d'individus possible

\begin{tabular}{|c|c|c|c|c|c|c|}
\hline $\begin{array}{l}\text { Lignes direc- } \\
\text { trices }\end{array}$ & Objectifs & ?ב & 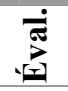 & Z & Description et justification & Pistes de bonification \\
\hline $\begin{array}{l}1 \text { - Possibilité } \\
\text { d'obtenir } \\
\text { l'usage du plus } \\
\text { grand nombre } \\
\text { de biens }\end{array}$ & $\begin{array}{l}\text { 1.2-Donner au } \\
\text { plus grand } \\
\text { nombre la } \\
\text { possibilité } \\
\text { d'utiliser des } \\
\text { biens indivi- } \\
\text { duels ou col- } \\
\text { lectifs }\end{array}$ & 3 & 0,9 & 2,7 & $\begin{array}{l}\text { Biens collectifs nombreux: cuisine, } \\
\text { salle à manger, salon avec télévi- } \\
\text { sion et système de son, salle } \\
\text { d'ordinateurs, salle d'exercices, } \\
\text { nourriture, jardin, piscine, aires de } \\
\text { repos, buanderie, bibliothèque... } \\
\text { Biens individuels; voitures, meu- } \\
\text { bles, effets personnels et chambres } \\
\text { louées. Les divers objets usagés } \\
\text { vendus à coût abordable permettent } \\
\text { un partage avec la population lo- } \\
\text { cale). }\end{array}$ & $\begin{array}{l}\text { Mettre des voitures } \\
\text { communes à la dispo- } \\
\text { sition des habitants. }\end{array}$ \\
\hline
\end{tabular}

Ganas qui, rappelons-le, ne se définit pas comme un écovillage mais s'est vu attribuer ici la même pondération au plan environnemental, présente un score significativement plus bas que les deux écovillages étudiés pour le pôle écologique. La communauté n'est pas dotée de politique où de norme de consommation verte concernant les achats (produits ménagers, nourriture et autres équipements). Les gestes à caractère écologique, s'ils sont posés, sont le fait de l'individu et non de la communauté. Ainsi, l'une des dimensions importantes de la grille, la dimension de planification, qui guide la mise en place des connaissances et des moyens pour réaliser ses aspirations en regard de l'environnement, explique en bonne partie le score plus bas obtenu par Ganas pour ce pôle. Toutefois, il est important de signaler que certains des choix de vie, même s'ils ne sont pas nécessairement guidés par des intentions écologiques, ont néanmoins un certain impact positif, notamment en ce qui a trait au partage des biens et à la possibilité d'utiliser les transports en commun pour ses déplacements courants. Par ailleurs, un autre aspect du développement durable peu considéré à Ganas et qui explique son faible score par rapport aux deux autres communautés est montré par l'objectif 4.1 .

Tableau 2 :

Ganas - Extrait du pôle écologique : maintenir les systèmes qui entretiennent la vie.

\begin{tabular}{|c|c|c|c|c|c|c|}
\hline $\begin{array}{l}\text { Lignes direc- } \\
\text { trices }\end{array}$ & Objectifs & อి & 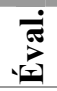 & Z & Description et justification & $\begin{array}{l}\text { Pistes de bonifica- } \\
\text { tion }\end{array}$ \\
\hline $\begin{array}{l}4 \text { - Maintien de } \\
\text { la biodiversité }\end{array}$ & $\begin{array}{l}4.1 \text { - Éva- } \\
\text { luer les } \\
\text { populations } \\
\text { des espèces } \\
\text { utilisées }\end{array}$ & 1 & 0,0 & 0,0 & $\begin{array}{l}\text { Connaissances, s'il y a, sont individuelles } \\
\text { et non communautaires. }\end{array}$ & $\begin{array}{l}\text { Être membre d'une } \\
\text { association ou d'un } \\
\text { groupe environne- } \\
\text { mentaliste. Organi- } \\
\text { ser des soirées thé- } \\
\text { matiques, des ate- } \\
\text { liers sur l'environ- } \\
\text { nement afin de } \\
\text { développer une } \\
\text { conscience mini- } \\
\text { male au sein des } \\
\text { habitants. }\end{array}$ \\
\hline
\end{tabular}




\section{Sirius}

Organisme à but non-lucratif, l'écovillage de Sirius a pour mission l'éducation à la vie communautaire, à l'écologie et à la spiritualité. Pour y parvenir, divers programmes, infrastructures et partenariats ont été mis sur pied au fil des années. Cette petite communauté de 35 habitants possède diverses installations, telles une salle de conférence et une cuisine munie d'équipements commerciaux. Plusieurs programmes sont offerts au grand public. Des chambres, des dortoirs et un camping permettent d'accommoder les participants des formations et activités d'apprentissage provenant de l'extérieur. Sirius entretient aussi une excellente relation avec le voisinage (surnommé à cet effet Earthstone village), qui participe régulièrement aux activités se déroulant sur le site de la communauté. Plusieurs de ces voisins sont membres de Sirius et utilisent certains services de la communauté, comme par exemple, une station d'huile végétale usagée (employée comme carburant pour des automobiles diesel converties à cette fin). Cette volonté évidente des habitants de partager leurs savoirs et leurs installations n'est certainement pas étrangère au pointage le plus élevé obtenu par Sirius pour le pôle équité $(85,5 \%)$, tel que le démontre l'objectif 3.4 de ce pôle.

Tableau 3 :

Sirius - Extrait du pôle équité: Équité entre les personnes, les communautés, les peuples et les générations

\begin{tabular}{|c|c|c|c|c|c|c|}
\hline $\begin{array}{l}\text { Lignes direc- } \\
\text { trices }\end{array}$ & Objectifs & $\stackrel{\Xi}{0}$ & 离 & है & Description et justification & Pistes de bonification \\
\hline $\begin{array}{l}3 \text {-Solidarité / } \\
\text { responsabilité / } \\
\text { imputabilité }\end{array}$ & $\begin{array}{l}3.4 \text { - Assu- } \\
\text { rer une } \\
\text { répartition } \\
\text { des avanta- } \\
\text { ges résultant } \\
\text { des projets } \\
\text { dans l'en- } \\
\text { semble de la } \\
\text { population }\end{array}$ & 2 & 1 & 2 & $\begin{array}{l}\text { Tenue de stages d'apprentissage, de } \\
\text { conférences, de retraites, de forma- } \\
\text { tions en méditation. Ouverture sur les } \\
\text { visiteurs et apprentis : chambres et } \\
\text { pensions à bas coût. Membership } \\
\text { parmi le voisinage élargi (Earthstone } \\
\text { Village). Partenariat avec l'Université } \\
\text { du Massachusette pour un cours ac- } \\
\text { crédité de permaculture chaque été sur } \\
\text { le site de la communauté. } \\
\text { Bénévolat pour des organismes de } \\
\text { Shutesbury }\end{array}$ & Aucune \\
\hline
\end{tabular}

Fort de ses 28 ans d'existence dans le cadre d'un mode de vie écologique, cet écovillage se révèle une source très inspirante en terme de comportement à faible impact écologique. Toutefois, cette démarche globale, quoique palpable concrètement sur le terrain, gagnerait, en regard des objectifs de la grille, à être articulée par une planification formelle, afin de déterminer le ratio productivité/utilisation des ressources renouvelables. Cette lacune en matière de planifi- cation formelle contribue à expliquer le fait que ce pôle n'a obtenu qu'un score moyen de 68,9 \%. L'examen de l'objectif 1.1 démontre en effet que Sirius pourrait optimiser son action en se dotant d'une meilleure planification et évaluation en matière de gestion environnementale. 


\section{Tableau 4 :}

\section{Sirius - Extrait du pôle écologique: Maintenir les systèmes qui entretiennent la vie}

\begin{tabular}{|c|c|c|c|c|c|c|c|}
\hline $\begin{array}{l}1 \text { - Utilisation } \\
\text { prioritaire des } \\
\text { ressources renou- } \\
\text { velables sous le } \\
\text { seuil de leur «re- } \\
\text { nouvelabilité » }\end{array}$ & $\begin{array}{l}1.1 \text { - Planifier } \\
\text { une utilisation } \\
\text { judicieuse des } \\
\text { ressources } \\
\text { renouvelables }\end{array}$ & 3 & 0,6 & & 1,8 & $\begin{array}{l}\text { Intention d'utiliser efficacement } \\
\text { les ressources traduites en actions } \\
\text { mais absence de plan formel. } \\
\text { Planification orale et aléatoire } \\
\text { durant les réunions hebdomadai- } \\
\text { res. } 70 \% \text { de l'énergie utilisée est } \\
\text { soit solaire passive (serres, mai- } \\
\text { sons dotées de fenêtres au sud), } \\
\text { solaire active (panneaux solaires) } \\
\text { ou éolienne. Système de récupé- } \\
\text { ration de l'eau de pluie pour } \\
\text { l'arrosage d'appoint du jardin. }\end{array}$ & $\begin{array}{l}\text { Gestion de l'eau à améliorer } \\
\text { eau potable et eaux grises - } \\
\text { système de purification des } \\
\text { eaux grises. Absence de plani- } \\
\text { fication "verte" globale pour } \\
\text { l'ensemble des ressources re- } \\
\text { nouvelables présentes sur le } \\
\text { terrain. Recours aux poêles de } \\
\text { masse pour chauffer les bâti- } \\
\text { ments permettrait d'utiliser } \\
\text { moins de bois. }\end{array}$ \\
\hline
\end{tabular}

\section{Earthaven}

Créé en 1995 dans l'esprit de devenir un lieu d'apprentissage et de démonstration à l'effet que l'organisation d'une société plus durable est possible, cet écovillage toujours en construction n'a pas encore atteint pleinement tous ces objectifs. Voilà qui explique en partie le fait que, pour tous les pôles, mis à part le pôle écologique, le score est plus faible que pour les autres cas de la présente étude. Le pôle social est celui qui présente le plus d'écart, avec 78,4\%. L'un des éléments les plus défavorables en regard des objectifs de ce pôle est le mode d'organisation territoriale décentralisée. Earthaven est constitué d'un centre, où l'on retrouve la salle du conseil, le magasin général et un restaurant-bar-bistro, autour duquel gravitent les voisinages (neighbourhoods). Bien que les réunions du conseil deux fois par mois et quelques activités sociales soient des occasions de rencontre, aucun système de communication et d'échange ne met en lien ces voisinages pour l'instant.

En outre, la situation géographique par rapport au grand centre urbain le plus proche, Asheville, situé à environ une heure de route, ne favorise pas les échanges d'informations et le dialogue entre les individus. En effet, ce manque de proximité physique limite la connectivité de la communauté avec l'extérieur. L'absence de transport en commun limite aussi d'emblée la venue de visiteurs et le rayonnement des actions de Earthaven. L'extrait du pôle social présenté dans le tableau 5 (objectif 3.1) montre l'un des effets de cette localisation et ce, malgré les efforts déployés pour dynamiser le milieu.

\section{Tableau 5 :}

Earthaven - Extrait du pôle social : Assurer à chaque être humain une vie saine et fournir des conditions qui permettront l'atteinte d'un sentiment d'harmonie personnel et une capacité de prendre en main son devenir

\begin{tabular}{|c|c|c|c|c|c|c|c|}
\hline $\begin{array}{l}\text { Lignes direc- } \\
\text { trices }\end{array}$ & Objectifs & อี & 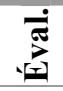 & $\frac{0}{0}$ & $\| \frac{\sqrt[c]{x}}{x}$ & Description et justification & Pistes de bonification \\
\hline $\begin{array}{l}3 \text { - Intégration } \\
\text { des individus à } \\
\text { la société par } \\
\text { une fonction } \\
\text { valorisante }\end{array}$ & $\begin{array}{l}3.1 \text { - Favo- } \\
\text { riser l'accès } \\
\text { à une oc- } \\
\text { cupation } \\
\text { pour cha- } \\
\text { cun }\end{array}$ & 3 & 0,6 & & 1,8 & $\begin{array}{l}\text { Quelques activités économiques: } \\
\text { camping, fabrication de remèdes } \\
\text { à base de plantes, Earthaven } \\
\text { Learning Center, pépinière etc. } \\
\text { Aucun soutien formel à l'entre- } \\
\text { preneuriat. } \\
\text { Localisation à près d'une heure } \\
\text { de la ville la plus près: moins }\end{array}$ & $\begin{array}{l}\text { Programme de soutien à } \\
\text { l'entrepreneuriat pour les } \\
\text { projets des membres. } \\
\text { Organisation d'une navette } \\
\text { journalière vers la ville et/ou } \\
\text { système formel de co- } \\
\text { voiturage. }\end{array}$ \\
\hline
\end{tabular}




\begin{tabular}{|c|c|c|c|c|c|c|}
\hline $\begin{array}{l}\text { Lignes direc- } \\
\text { trices }\end{array}$ & Objectifs & อ & 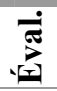 & ¿ & Description et justification & Pistes de bonification \\
\hline & & & & & $\begin{array}{l}\text { d'opportunités d'emplois pour les } \\
\text { travailleurs, plus difficile } \\
\text { d'établir sur place des entreprises } \\
\text { de services (faute de marché). } \\
\text { Tenue d'activités diversifiées } \\
\text { permettant à tous de participer } \\
\text { selon leurs talents et intérêts. }\end{array}$ & $\begin{array}{l}\text { «Point de service en ville»: } \\
\text { point de dépôt ou de vente } \\
\text { pour les biens produits dans } \\
\text { l'écovillage; quelques cham- } \\
\text { bres pour loger temporaire- } \\
\text { ment membres et visiteurs. }\end{array}$ \\
\hline
\end{tabular}

Véritable champion de la décroissance viable, cet écovillage est incontestablement engagé dans la voie du respect de l'environnement et de ses écosystèmes. Dès le début du projet, les fondateurs ont étudié le terrain et ont planifié l'utilisation des ressources naturelles qui s'y trouvaient. En effet, quatre plans (foresterie, gestion de l'eau, énergie et aménagement d'ensemble) encadrent et guident les actions des habitants de Earthaven. Par contre, quelques éléments restent encore à améliorer, par exemple, l'utilisation de l'automobile, encore trop sur une base individuelle. Le score pour le pôle écologique (76\%) est néanmoins le plus élevé des trois cas étudiés.

Tableau 6 :

Earthaven - Extrait du pôle écologique: Maintenir les systèmes qui entretiennent la vie

\begin{tabular}{|c|c|c|c|c|c|c|}
\hline $\begin{array}{l}\text { Lignes directri- } \\
\text { ces }\end{array}$ & Objectifs & ?. & 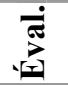 & 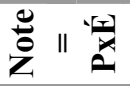 & Description et justification & Pistes de bonification \\
\hline $\begin{array}{l}2 \text { - Utilisation } \\
\text { judicieuse des } \\
\text { ressources non- } \\
\text { renouvelables }\end{array}$ & $\begin{array}{l}2.5 \text { - Plani- } \\
\text { fier une } \\
\text { utilisation } \\
\text { judicieuse } \\
\text { de l'énergie }\end{array}$ & 3 & 0,8 & 2,4 & $\begin{array}{l}\text { Recours à des sources d'énergies } \\
\text { renouvelables seulement. Résidences } \\
\text { personnelles possédant toutes des } \\
\text { panneaux solaires et les bâtisses } \\
\text { communautaires sont alimentées par } \\
\text { micro-hydroélectricité. Responsabili- } \\
\text { sation des écovillageois face à leur } \\
\text { consommation d'énergie (évitent le } \\
\text { gaspillage). Réfrigérateurs horizon- } \\
\text { taux (éconoénergitique : lorsque ou- } \\
\text { vert, l'air froid ne s'échappe pas). } \\
\text { Caveau à légumes pour chaque mai- } \\
\text { son. }\end{array}$ & $\begin{array}{l}\text { Recours à une alterna- } \\
\text { tive pour le fonctionne- } \\
\text { ment des automobiles } \\
\text { (biodiesel). Covoiturage } \\
\text { à améliorer: système de } \\
\text { communication formelle } \\
\text { devrait être établi afin de } \\
\text { mettre en contact les } \\
\text { voyageurs et automobi- } \\
\text { listes. }\end{array}$ \\
\hline
\end{tabular}

\section{Conclusion}

La démarche exploratoire auprès de ces trois communautés visait à vérifier la capacité de certains écovillages à répondre aux impératifs du développement durable dans leur fonctionnement concret. Elle n'avait pas la prétention de répondre à toutes les questions d'ordre théorique ou sociologique mais cherchait simplement à ouvrir une porte sur des recherches à approfondir quant aux stratégies innovantes de développement durable applicables en contexte communautaire. Le recours à la grille d'analyse de développement durable a permis de dégager divers éléments qu'il importe de considérer en regard aux exigences du développement durable.

En plus d'être des lieux propices à l'innovation et à l'originalité, les écovillages donnent à l'individu la possibilité de se réapproprier la communauté comme noyau de rapports humains et comme catalyseur d'alternatives de vie juste et viable. Au plan écologique, ces communautés présentent des scores différents, les points forts ou faibles variant en fonction de leurs objectifs spécifiques. On constate toutefois que l'innovation en matière d'alternative écologique porte fruit dans les deux écovillages lors- 
qu'on les compare avec Ganas, une communauté intentionnelle témoin. Cependant, les objectifs écologiques semblent plus difficiles à atteindre. Il s'agit d'une importante piste de recherche à explorer au sujet des écovillages mais aussi à l'égard du développement durable lui-même et de sa complexité d'application. Finalement, le volet économique, lorsqu'il est bien intégré, incite à un certain dynamisme interne et maintient le contact avec l'extérieur.

Les expériences de Sirius et Earthaven prouvent que l'écovillage n'est pas un concept flou, l'appellation pouvant effectivement correspondre à des initiatives dont les objectifs et leur mise en œuvre tendent bel et bien vers le développement durable. L'analyse des résultats montre que le mode de vie de ces écovillageois qui, au plan social et économique, répond aux besoins de base et améliore la qualité de vie, est réaliste et possible et ce, même en déployant des efforts soutenus pour préserver la capacité de portée de la nature. Même s'ils sont loin d'être une panacée, les écovillages présentent de nombreuses facettes qui méritent une attention particulière en regard de la recherche de solutions pragmatiques à des problèmes environnementaux. Il faut maintenant chercher à dégager le potentiel de ces pratiques pour qu'elles puissent devenir de véritables leviers en vue d'une prise de conscience et d'actions plus globales.

\section{Les expériences de Sirius et Earthaven prou- vent que l'écovillage n'est pas un concept flou, l'appellation pouvant effectivement correspondre à des initiatives dont les objec- tifs et leur mise en œuvre tendent bel et bien vers le développement durable.}

\section{Notes et références}

1 Wakernagel, M. et W. Rees (1999). Notre empreinte écologique, Montréal, Éditions Écosociété.
2 Pour de plus amples informations sur le concept, consultez l'article «Regard neuf sur le monde rural dans tous ses états » paru dans Organisation et territoires, vol. 14 , no. 3 .

3 Ministère du Développement durable, Environnement et Parc, www.mddep.gouv.qc.ca

4 Gaia Trust est une association charitable danoise fondée en 1987 par Ross et Hildur Jackson. Leur intention était de soutenir des initiatives porteuses de valeurs menant à une société viable et plus spirituelle. Pour plus d'informations : http://www.gaia.org/

5 Le GEN est une association mondiale de personnes et de groupes qui rassemblement et partagent leurs idées, promeut des technologies douces et développent des liens entre les écovillages par le biais d'annuaires et de bulletins culturels et éducatifs. Elle vise à mettre en œuvre le développement durable au sein des communautés. Pour plus d'informations : http://www.gen.ecovillage.org

6 Le réseau mondial des écovillages: http://gen.ecovillages.org

7 Le réseau des écovillages du Canada: http://enc.ecovillage.org

8 Villeneuve, C., (1999, révisé 2004) Comment réaliser une analyse de développement durable? Département des sciences fondamentales, Université du Québec à Chicoutimi

9 Communauté intentionnelle est un terme inclusif qui comprend des sous-ensembles de communautés tel les écovillages, les coopératives de logement, le cohousing, etc. Voir le site de «Fellowship for Intentional community », www.ic.org pour un répertoire de ces communautés ou pour plus d'informations.

10 Pour plus d'informations, visitez leur site web à l'adresse : www.ganas.org

11 Pour plus d'informations, visitez le site web de Sirius Community à l'adresse : www.siriuscommunity.org

12 Pour plus d'informations, visitez le site web de Earthaven Ecovillage à l'adresse : www.earthaven.org 